\title{
Diagnosis, Causes and Treatments of Instability Following Total Knee Arthroplasty
}

\author{
Moon Jong Chang, MD, Hyungtae Lim, MD, Na Rae Lee, and Young-Wan Moon, MD \\ Department of Orthopedic Surgery, Samsung Medical Center, Sungkyunkwan University School of Medicine, Seoul, Korea
}

\begin{abstract}
Instability following total knee arthroplasty is one of the major causes of revision surgery. In most cases, it can be prevented by using an appropriate prosthesis and a good surgical technique. Particular attention should be given to confirmation of diagnosis for which thorough history taking, complete physical examination and radiographic evaluation are needed. With regard to treatment, identification of the etiology of instability is crucial for establishing proper treatment plans; instability would persist without correction of the cause of the initial instability. For successful revision surgery, balanced medio-lateral and flexion-extension gaps should be achieved. Constrained or rotating-hinge total knee prosthesis should also be considered as an alternative option for certain subsets of patients with instability.
\end{abstract}

Keywords: Knee, Arthroplasty, Instability, Prosthesis failure

\section{Introduction}

Total knee arthropalsty (TKA) has demonstrated superior efficacy in terms of pain relief and functional improvement, and the 10 -year survival rates of the surgical procedure have been reported to be $\geq 90 \%{ }^{1,2)}$. In the meantime, however, revision procedures have been performed with increasing frequency. In the US, revision TKA that has grown in prevalence, as well as primary TKA, consisted approximately $10 \%$ of the total TKA cases in $2002^{3)}$. In South Korea, the incidence of primary TKA increased by $407 \%$ between 2001 and $2010^{4}$ and has been rising steadily thereafter. Although revision TKA has been considered necessary in only $2 \%-3.1 \%$ of the patients after primary $\mathrm{TKA}^{4,5)}$, it seems reasonable to expect that the increasing volume of primary TKA will

Received December 5, 2013; Revised January 2, 2014;

Accepted February 24, 2014

Correspondence to: Young-Wan Moon, MD

Department of Orthopedic Surgery, Samsung Medical Center,

Sungkyunkwan University School of Medicine, 81 Irwon-ro, Gangnamgu, Seoul 135-710, Korea

Tel: +82-2-3410-3533, Fax: +82-2-3410-0061

E-mail: ywmoon@skku.edu

This is an Open Access article distributed under the terms of the Creative Common Attribution Non-Commercial License (http://creativecommons.org/licenses/by-nc/3.0/) which permits unrestricted non-commercial use, distribution, and reproduction in any medium, provided the original work is properly cited. correlate into higher revision rates ${ }^{4}$.

Postoperative instability is one of the major causes of revision TKA that may be required for various reasons, including polyethylene wear, metal wear, implant loosening, instability, infection, malposition, periprosthetic fracture, and implant breakage ${ }^{6-8)}$. Instability is the third most common mode of TKA failure that necessitates a revision procedure in $7.3 \%-28.9 \%$ of the cases ${ }^{9-12)}$. Although prevention is the best medicine, once instability occurs following TKA, the first steps of treatment are accurate diagnosis and identification of the exact cause of instability. Symptomfocused treatments that leave the underlying causes unaddressed often carry the risk of recurrence.

In this review article, we will discuss the diagnosis, causes, and treatments of instability following TKA.

\section{Diagnosis}

\section{Clinical Evaluation}

Instability following TKA can be defined as abnormal and excessive displacement of a knee prosthesis accompanied by clinical failure $^{13,14)}$. Rheumatoid arthritis, connective tissue diseases, severe osteoporosis, and neuropathy are risk factors for postoperative instability. Correction procedures for severe deformity, such as aggressive ligament releases, are also related ${ }^{15)}$. The instability can be attributable to neuromuscular pathology ${ }^{16,17)}$ : recurvatum is predisposed by quadriceps weakness and medial thrust, by hip www.jksrr.org 


\section{Chang et al. Diagnosis, Causes and Treatments of Instability Following Knee Arthroplasty}

abductor weakness ${ }^{15}$. Obesity has also been associated with the increased risk of instability because collateral ligament injuries tend to occur due to the difficulty of surgical exposure. In a study by Winiarsky et al. ${ }^{18)}$, medial collateral ligament (MCL) avulsion was observed in approximately $8 \%$ of the total TKA patients ${ }^{18}$.

Instability following TKA can be suspected or diagnosed with medical history taking, examinations for symptoms, and physical tests. Medical history taking should particularly focus on the diagnosis for the primary TKA, the degree of preoperative deformity or joint contracture, previous surgery on the ipsilateral knee, surgical techniques and type of prosthesis used in primary TKA, rehabilitation regimen, and history of trauma after primary TKA $^{14)}$. Symptoms of instability vary, ranging from subtle sense of instability to dislocation. Instability can be suspected on the basis of the following symptoms: stiff-legged gait, hyperextension of the knee to lock the joint during stance phase, varus or valgus thrust gait, and abnormal or rotated foot progression angle during walking ${ }^{19}$. Patients with flexion instability may have difficulty in climbing up and down stairs, present with recurrent joint effusions, or complain of diffuse periretinacular tenderness or pain in the tendinous attachment site ${ }^{20)}$. It is advised to exclude other causes, such as infection, and identify the cause that is associated with secondary instability, such as component loosening. Basic laboratory tests, including white blood cell count, erythrocyte sedimentation rate, and C-reactive protein, should be performed to rule out infection ${ }^{21}$. Recently, Aggarwal et al. ${ }^{22)}$ introduced a new leukocyte esterase test that allows for easy detection of infection using joint fluid with high sensitivity and specificity ${ }^{22}$.

On physical examination, varus-valgus laxity is assessed with the knee in extension and $30^{\circ}$ flexion. If flexion instability is present, anterior drawing of the knee from $90^{\circ}$ flexion with the patient in a seated position keeping the foot flat on the ground elicits excessive motion or symptoms consistent with the patient's complaints $^{23}$. Mid-flexion instability can be indicated by stability in full extension and $90^{\circ}$ flexion and instability in $30^{\circ}-45^{\circ}$ flexion $^{24)}$.

\section{Radiographic Evaluation}

In general, radiographic follow-up after TKA involves fulllength standing anteroposterior (AP), lateral, and skyline radiographs. The AP and lateral views are taken with the $\mathrm{X}$-ray beam directed parallel to the base plate of the tibial component to identify any changes between bone cement and prosthesis. Weightbearing radiographs are for identification of asymmetric wear or failure of the polyethylene liner ${ }^{19)}$ as well as lower limb malalignment that causes or worsens the instability, component malposition, and loosening. Skyline views facilitate observation of the location of the patella in the femoral groove, patellar subluxation, and status of the patellar component. Varus-valgus stress views allow assessment of the degree of ligament laxity, providing a more objective indicator of the presence of instability: significant lift off under application of stress indicates presence of medial or lateral instability ${ }^{21)}$ (Fig. 1). In the absence of specific numerical criteria for radiographic diagnosis of instability following TKA, a failure of primary TKA should be determined based on findings from serial X-rays.

Computed tomography is more effective in visualizing component loosening associated with secondary instability. It is also useful for identifying mal-rotation of the femoral component that can result in an asymmetric flexion space or, in particular, patel-
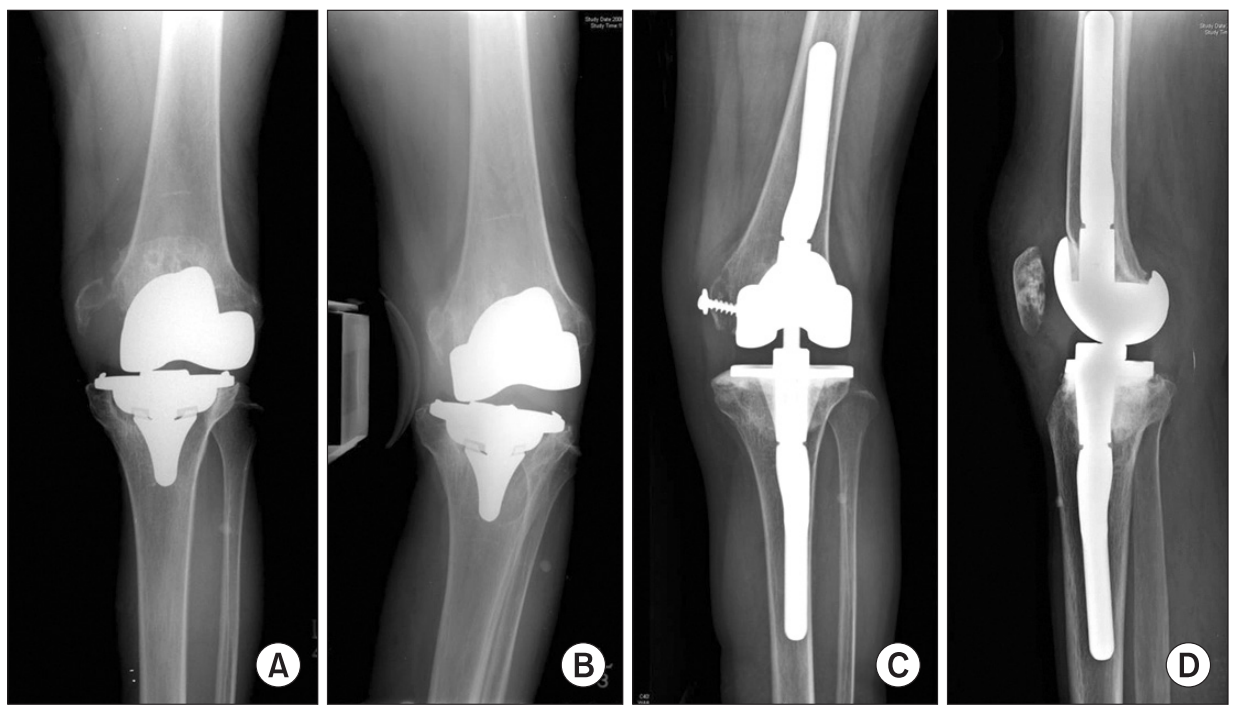

Fig. 1. Varus-valgus stress radiographs of the knee with instability showing medial femoral condyle avulsion fracture caused by severe osteolysis (A) and lift off in the lateral compartment due to combined lateral laxity (B). (C, D) Revision total knee arthroplasty was performed using a constrained condylar implant. 
lar instability ${ }^{3)}$.

\section{Causes and Treatments}

Instability can be prevented with appropriate prosthesis selection and a good surgical technique in most cases ${ }^{13}$. Prevention is the best treatment with regard to instability following TKA. Thus, the importance of flexion-extension and medio-lateral gap balancing should never be underestimated.

A deep MCL release is effective for gap balancing for most varus deformities, and subperiosteal release of the MCL can be a solution for severe varus deformities ${ }^{25}$. However, a sequential release of the posteromedial capsule, semimembranosus tendon, superficial MCL, and the pes anserinus may be required for otherwise intractable varus deformities. In such a case, the procedure for varus deformity correction should be performed with care not to cause excessive opening of the medial space in flexion after complete release of the MCL.

In the valgus knee, contracture of the lateral soft tissue structures, including the lateral collateral ligament (LCL), iliotibial band (ITB), and lateral capsule, is accompanied by gradual attenuation of medial soft tissues. Structures that may need to be released for valgus deformity correction include the lateral capsule, LCL, arcuate ligament, popliteus tendon, lateral femoral periosteum, and distal ITB, and the adjacent lateral intermuscular septum can be partially released from the bony attachment site. The ITB can be released from the Gerdy's tubercle and the rest, from the lateral femoral condyle. The ITB is the first to be released if the knee is tight laterally in extension. Releases of the posterolateral capsule and the LCL are performed for tightness in both flexion and extension. The popliteus tendon release is useful for tightness of the lateral flexion gap ${ }^{26)}$. The pie crusting technique using stab incisions should be employed for valgus deformity correction to avoid the risk of posterolateral instability due to excessive soft tissue stripping in the lateral femoral condyle $e^{27,28)}$. For severe valgus deformities, medial ligament advancement or reconstruction may be required due to MCL laxity ${ }^{29,30)}$. In order to prevent leglengthening and joint line elevation after deformity correction, excessive ligament releases should be avoided. In addition, care should be exercised to avoid the risk of neural damage caused by stretching of the peroneal nerve in elderly patients with $\geq 20^{\circ}$ of valgus deformity ${ }^{31,32)}$.

In revision TKA, it is imperative to identify the cause of TKA failure and solve the specific problems with appropriate treatment methods. Instability is mostly attributable to component loosening, bone loss, prosthetic breakage, improper component size or position, periprosthetic fracture, wear, or collateral ligament laxity $^{15)}$. Restoration of the mechanical limb alignment is essential for successful revision TKA ${ }^{15}$. In addition, assessment on the ligament integrity and medio-lateral flexion-extension gap balancing should be performed ${ }^{15}$. However, soft tissue structures that were clearly visible in primary TKA may become thicker, weakened, transformed into scar tissues, or even disappear at the time of revision surgery. The loss of soft tissue support and deformation make gap balancing difficult. For varus-valgus balancing, the contracted portions of the medial or lateral ligament or tendon should be released, whereas an attenuated ligament may need to be treated with advancement or reconstruction. Gap balance is also affected by releases of the contracted posterior soft tissues, bone resection level, and component position and size. It is recommended to use a minimally constrained type of prosthesis to maintain stability ${ }^{33,34}$. In most cases, it is practically impossible to achieve stability without implanting a constrained prosthesis in unstable knees that require ligament reconstruction ${ }^{35}$ (Fig. 1). However, constrained condylar implants have limited usage among relatively young and active patients because they have been associated with increased risk of revision TKA due to early loosening caused by excessive stress on the fixation interface ${ }^{32}$.

Instability following TKA can be divided into different types according to classification criteria and different treatment regimens are prescribed depending on the type of instability. Vince et al. ${ }^{15)}$ suggested there are 1) varus/valgus, 2) recurvatum, 3) AP (in flexion), and 4) global types of instability according to the direction of deforming force. Parratte and Pagnano ${ }^{14)}$ proposed three different types of instability: 1) extension instability, 2) flexion instability, and 3) genu recurvatum.

\section{Extension Instability}

Extension instability can be broadly divided into symmetric extension instability and asymmetric extension instability ${ }^{14)}$. An excessive bone resection may result in symmetric extension instability. Care should be taken not to cause joint line elevation by minimizing bone resection when the amount of distal femur cut is greater than the distal thickness of the femoral component. Considering that the posterior capsule and ligament play a major role in the extension gap, proper soft tissue balancing and a posterior release should precede an additional bone resection. Extensive bone loss in the distal femur cannot simply be managed with the placement of a thick polyethylene liner due to the risk of joint line elevation and excessive tightness in flexion. A joint line elevation results in abnormal knee kinematics, limiting range of flexion and patellar function and causing instability in $30^{\circ}-45^{\circ}$ 


\section{Chang et al. Diagnosis, Causes and Treatments of Instability Following Knee Arthroplasty}

mid-flexion, in spite of stability in extension and $90^{\circ}$ flexion. In such a case, augmentation of the distal femur is required for joint line restoration and extension gap adjustment, for flexion/extension gap balance.

Asymmetric extension instability can be encountered more often in clinical settings. It is mainly associated with mediolateral gap imbalance and may become worse in the presence of malalignment. It can also occur due to traumatic collateral ligament injury following successful TKA. The most common cause of asymmetric extension instability is insufficient correction of deformity. Therefore, a desired limb alignment and medio-lateral gap balance should be achieved in primary TKA using a proper soft tissue release. The use of a constrained condylar implant can be considered as an option in primary TKA, if gap imbalance appears to persist due to severe deformity that limits the efficacy of a soft tissue release or an iatrogenic injury to the collateral ligament occurs during surgery ${ }^{32,36)}$.

\section{Flexion Instability}

Flexion instability can be defined as the presence of the flexion gap larger than the extension gap. It occurs mostly due to an undersized femoral component or a steep tibial slope. The use of an undersized femoral component with a decreased AP dimension in revision TKA due to excessive bone loss leads to an increase in the flexion gap. Therefore, the decision on the femoral component size should be based not on the remaining bone stock but on the assessment of flexion stability. During revision TKA, efforts should be made to replicate the original anatomy of the distal femur, for which the size of the removed femoral component and the radiographs of the other knee can be referred to. If the flex- ion gap is smaller than the extension gap, a femoral component smaller than the original can be effective for enlarging the flexion gap; however, a greater femoral component should be implanted, if instability is present in flexion. Therefore, in case of extensive bone loss, aggressive augmentation using a metal or bone graft should be performed for flexion gap restoration. Malrotation of the femoral component can be another cause for flexion instability. Excessive internal rotation of the original femoral component results in extensive resection of the postero-lateral femoral condyle and excessive external rotation, extensive resection of the postero-medial femoral condyle, eventually leading to creation of a trapezoidal flexion gap. Thus, care should be taken to the rotational alignment of the femoral component in revision TKA. The transepicondylar axis, if it remains in the revision surgery, serves as the best reference for accurate restoration of the rotational alignment that should be parallel to the transepicondylar axis.

Flexion instability can occur in patients with PCL-retaining implants who have lax or injured PCL. In these patients, the resultant flexion gap of an inappropriately great size causes flexion instability and even dislocation in severe cases, which can be treated with use of a posterior stabilized implant in revision TKA (Fig. 2). Recent posterior stabilized implants are devised to have a longer jump distance, the distance for the cam to jump over the post, making dislocation a very rare event. However, if varusvalgus instability is present, even these implants may not provide sufficient stability to prevent dislocation.

Flexion instability is commonly accompanied by collateral ligament laxity, which requires the use of a constrained type implant. If a dislocation occurs, it is initially treated with closed reduction, a brace, and avoidance of positions that can cause dislocation.
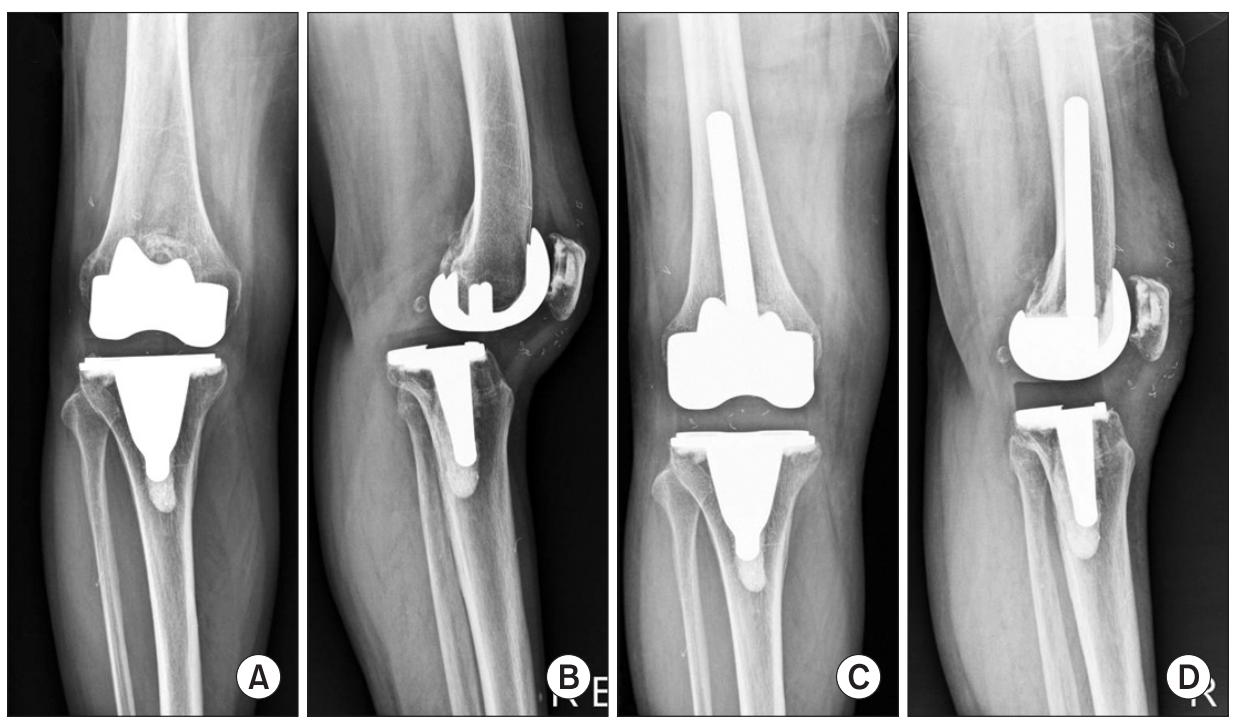

Fig. 2. (A, B) Radiographs of the knee with instability after total knee arthroplasty using a cruciate-retaining knee implant. (C, D) Conversion of the femoral component to a posterior stabilized femoral component was performed in revision sugery. 

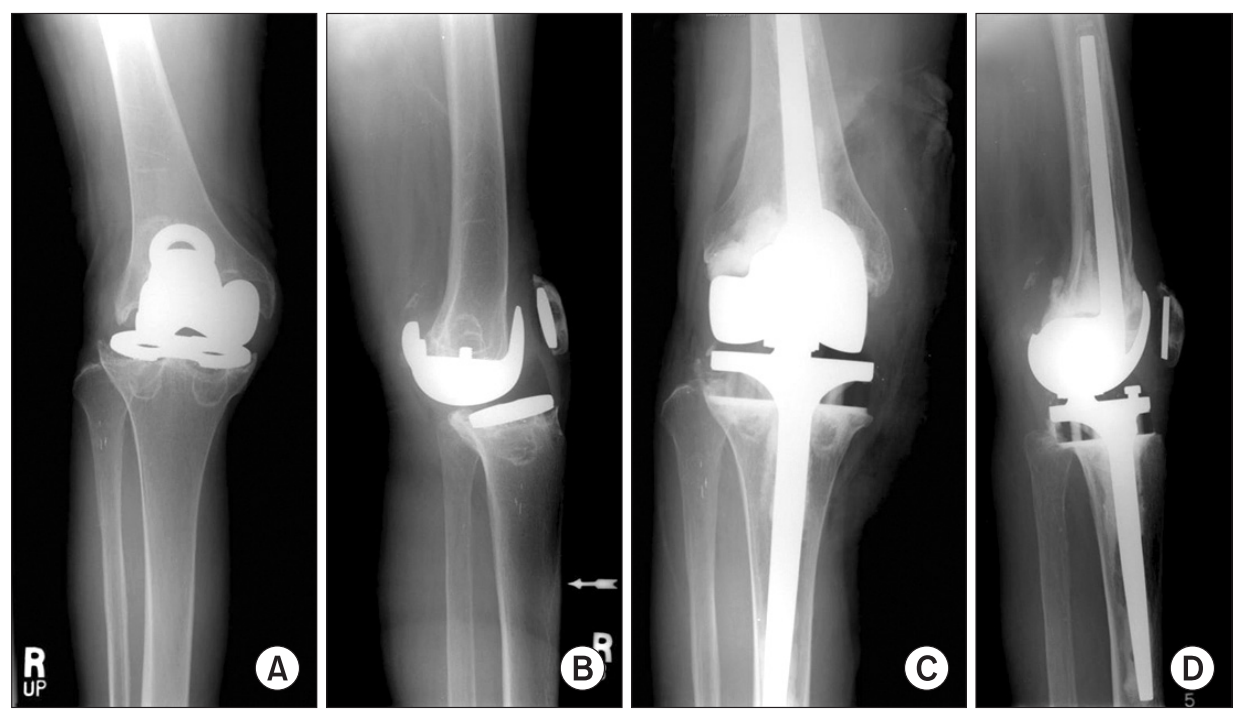

Fig. 3. Radiographs of the knee with severe bone loss and osteolysis combined with recurvatum (A) and global instability (B). (C, D) Revision surgery was performed using a rotating hinge knee prosthesis.

However, if it recurs, surgical intervention becomes unavoidable. If the extension gap allows, insertion of a thicker polyethylene insert may be tried to obtain stability. However, if flexion instability persists, metal augmentation should be performed to compensate for the flexion gap in revision TKA and the use of a constrained condylar implant should be considered ${ }^{37)}$. A rotatinghinge total knee prosthesis should be viewed as an option when stability is not attainable with a constrained condylar implant due to extensive soft tissue laxity in the knee.

\section{Genu Recurvatum}

Recurvatum following TKA is one of the most challenging deformities. Thus, prevention is the best treatment ${ }^{14)}$. There are some surgical techniques designed to prevent recurvatum during primary TKA in patients with hyperextension deformity: underresection of the distal femur and insertion of an augmentation block in the femoral component to make some degree of flexion contracture remain after surgery ${ }^{14)}$. Krackow and Weiss ${ }^{17)}$ suggested proximal or posterior placement of the femoral origins of the MCL and LCL to maintain normal tension with the knee in full extension.

Recurvatum after TKA may require the use of a brace for life or a rotating hinge total knee prosthesis, or arthrodesis ${ }^{24)}$ (Fig. 3). Rotating hinge total knee prosthesis that is rarely used due to the risk of aseptic loosening may be useful for recurvatum following TKA.

\section{Conclusions}

TKA failure resulting from instability can be prevented with a proper selection of prosthesis and surgical technique. Treatment success of instability following TKA is primarily dependent on the accurate diagnosis. Thus, thorough history taking and physical examination are essential, considering symptoms can be as mild as pain or vague sense of instability. It is imperative to identify the root cause of instability to determine proper treatment. During revision TKA, care should be taken to medio-lateral soft tissue balancing, flexion-extension gap equalization, and restoration of stability with proper selection of a constrained implant.

\section{Conflict of Interest}

No potential conflict of interest relevant to this article was reported.

\section{References}

1. Choi YJ, Lee KW, Kim CH, Ahn HS, Hwang JK, Kang JH, Han HD, Cho WJ, Park JS. Long-term results of hybrid total knee arthroplasty: minimum 10-years follow-up. Knee Surg Relat Res. 2012;24:79-84.

2. Patil SS, Branovacki G, Martin MR, Pulido PA, Levy YD, Colwell CW Jr. 14-year median follow-up using the press-fit condylar sigma design for total knee arthroplasty. J Arthroplasty. 2013;28:1286-90.

3. Math KR, Zaidi SF, Petchprapa C, Harwin SF. Imaging of total knee arthroplasty. Semin Musculoskelet Radiol. 2006;10:47-63.

4. Koh IJ, Kim TK, Chang CB, Cho HJ, In Y. Trends in use of total knee arthroplasty in Korea from 2001 to 2010. Clin Or- 
thop Relat Res. 2013;471:1441-50.

5. Koh IJ, Cho WS, Choi NY, Kim TK; Kleos Korea Research Group. Causes, risk factors, and trends in failures after TKA in Korea over the past 5 years: a multicenter study. Clin Orthop Relat Res. 2014;472:316-26.

6. Bozic KJ, Kurtz SM, Lau E, Ong K, Chiu V, Vail TP, Rubash HE, Berry DJ. The epidemiology of revision total knee arthroplasty in the United States. Clin Orthop Relat Res. 2010;468:45-51.

7. Sharkey PF, Hozack WJ, Rothman RH, Shastri S, Jacoby SM. Insall Award paper. Why are total knee arthroplasties failing today? Clin Orthop Relat Res. 2002;(404):7-13.

8. Gioe TJ, Killeen KK, Grimm K, Mehle S, Scheltema K. Why are total knee replacements revised?: analysis of early revision in a community knee implant registry. Clin Orthop Relat Res. 2004;(428):100-6.

9. Mulhall KJ, Ghomrawi HM, Scully S, Callaghan JJ, Saleh KJ. Current etiologies and modes of failure in total knee arthroplasty revision. Clin Orthop Relat Res. 2006;446:45-50.

10. Tay KS, Lo NN, Yeo SJ, Chia SL, Tay DK, Chin PL. Revision total knee arthroplasty: causes and outcomes. Ann Acad Med Singapore. 2013;42:178-83.

11. Suarez J, Griffin W, Springer B, Fehring T, Mason JB, Odum S. Why do revision knee arthroplasties fail? J Arthroplasty. 2008;23(6 Suppl 1):99-103.

12. Fehring TK, Valadie AL. Knee instability after total knee arthroplasty. Clin Orthop Relat Res. 1994;(299):157-62.

13. Rodriguez-Merchan EC. Instability following total knee arthroplasty. HSS J. 2011;7:273-8.

14. Parratte S, Pagnano MW. Instability after total knee arthroplasty. Instr Course Lect. 2008;57:295-304.

15. Vince KG, Abdeen A, Sugimori T. The unstable total knee arthroplasty: causes and cures. J Arthroplasty. 2006;21(4 Suppl 1):44-9.

16. Meding JB, Keating EM, Ritter MA, Faris PM, Berend ME. Genu recurvatum in total knee replacement. Clin Orthop Relat Res. 2003;(416):64-7.

17. Krackow KA, Weiss AP. Recurvatum deformity complicating performance of total knee arthroplasty: a brief note. J Bone Joint Surg Am. 1990;72:268-71.

18. Winiarsky R, Barth P, Lotke P. Total knee arthroplasty in morbidly obese patients. J Bone Joint Surg Am. 1998;80: 1770-4.

19. Gonzalez MH, Mekhail AO. The failed total knee arthroplasty: evaluation and etiology. J Am Acad Orthop Surg. 2004;12:436-46.
20. Schwab JH, Haidukewych GJ, Hanssen AD, Jacofsky DJ, Pagnano MW. Flexion instability without dislocation after posterior stabilized total knees. Clin Orthop Relat Res. 2005;440:96-100.

21. Hofmann S, Seitlinger G, Djahani O, Pietsch M. The painful knee after TKA: a diagnostic algorithm for failure analysis. Knee Surg Sports Traumatol Arthrosc. 2011;19:1442-52.

22. Aggarwal VK, Tischler E, Ghanem E, Parvizi J. Leukocyte esterase from synovial fluid aspirate: a technical note. J Arthroplasty. 2013;28:193-5.

23. Pagnano MW, Hanssen AD, Lewallen DG, Stuart MJ. Flexion instability after primary posterior cruciate retaining total knee arthroplasty. Clin Orthop Relat Res. 1998;(356):39-46.

24. Del Gaizo DJ, Della Valle CJ. Instability in primary total knee arthroplasty. Orthopedics. 2011;34:e519-21.

25. Insall JN, Binazzi R, Soudry M, Mestriner LA. Total knee arthroplasty. Clin Orthop Relat Res. 1985;(192):13-22.

26. Laskin RS. Total knee replacement. London: Springer; 1991. p41-53.

27. Mihalko WM, Krackow KA. Anatomic and biomechanical aspects of pie crusting posterolateral structures for valgus deformity correction in total knee arthroplasty: a cadaveric study. J Arthroplasty. 2000;15:347-53.

28. Aglietti P, Lup D, Cuomo P, Baldini A, De Luca L. Total knee arthroplasty using a pie-crusting technique for valgus deformity. Clin Orthop Relat Res. 2007;464:73-7.

29. Favorito PJ, Mihalko WM, Krackow KA. Total knee arthroplasty in the valgus knee. J Am Acad Orthop Surg. 2002;10:16-24.

30. Krackow KA, Holtgrewe JL. Experience with a new technique for managing severely overcorrected valgus high tibial osteotomy at total knee arthroplasty. Clin Orthop Relat Res. 1990;(258):213-24.

31. Clarke HD, Fuchs R, Scuderi GR, Scott WN, Insall JN. Clinical results in valgus total knee arthroplasty with the "pie crust" technique of lateral soft tissue releases. J Arthroplasty. 2005;20:1010-4.

32. Easley ME, Insall JN, Scuderi GR, Bullek DD. Primary constrained condylar knee arthroplasty for the arthritic valgus knee. Clin Orthop Relat Res. 2000;(380):58-64.

33. Bugbee WD, Ammeen DJ, Engh GA. Does implant selection affect outcome of revision knee arthroplasty? J Arthroplasty. 2001;16:581-5.

34. Scuderi GR. Revision total knee arthroplasty: how much constraint is enough? Clin Orthop Relat Res. 2001;(392):3005. 
35. Pritsch M, Fitzgerald RH Jr, Bryan RS. Surgical treatment of ligamentous instability after total knee arthroplasty. Arch Orthop Trauma Surg. 1984;102:154-8.

36. Leopold SS, McStay C, Klafeta K, Jacobs JJ, Berger RA, Rosenberg AG. Primary repair of intraoperative disruption of the medical collateral ligament during total knee arthroplasty. J Bone Joint Surg Am. 2001;83:86-91.

37. Nelson CL, Gioe TJ, Cheng EY, Thompson RC Jr. Implant selection in revision total knee arthroplasty. J Bone Joint Surg Am. 2003;85 Suppl 1:S43-51. 